\title{
ON THE ORDERING OF THE NONSTANDARD REAL LINE
}

\author{
MAURO DI NASSO AND MARCO FORTI
}

\begin{abstract}
We investigate order-theoretic properties of the nonstandard real line, by isolating the basic order-types involved, and the possible relations among them. We focus on order-isomorphisms between the infinitesimals, the infinites, and the whole hyperreal line. We also compute the main cardinal invariants of the order-topology of the nonstandard real line.
\end{abstract}

\section{INTRODUCTION}

An interest in the structural properties of the nonstandard (or hyper-) real line $* \mathbb{R}$ arose since the beginnings of nonstandard analysis. In the seminal paper [30], E. Zakon extensively studied $* \mathbb{R}$, both from the viewpoint of ordering and that of topology. Some relevant problems were left open, and in the following years many papers appeared were the subject was further investigated (see e.g. [23], [19], [17], [11], [9], [7], [21], [13], and [28]).

In this paper we concentrate on the order-theoretic side. We isolate the basic order-types originated by the structure of ${ }^{*} \mathbb{R}$, and we study the possible relations among them. In particular, some implications will be shown not to hold in general by constructing suitable models as counter-examples.

The notion of hyperreal line we are considering here follows the usual "external" viewpoint to nonstandard analysis, e.g. as given by the so-called superstructure approach. ${ }^{1}$ In a general set-theoretic setting, all the results given here about the hyperreal line, could be reformulated as (external) properties of the real line in some elementary extension of $V_{\omega+\omega}$, the "true" model of ZFC - Replacement. However, for convenience, in this paper we shall stick to the most popular superstructure approach to nonstandard analysis. Let us briefly recall the basics of this approach.

The superstructure over a set $X$ is the union $V(X)=\bigcup_{n \in \mathbb{N}} V_{n}(X)$ where $V_{0}(X)=$ $X$, and $V_{n+1}(X)=V_{n}(X) \cup \wp\left(V_{n}(X)\right)$, where $\wp$ is the powerset operator. The standard model of analysis is the superstructure $V(\mathbb{R})$. A nonstandard model of analysis is a superstructure $V\left({ }^{*} \mathbb{R}\right)$ which is a bounded elementary extension of the standard model via a nonstandard embedding denoted by the symbol $*$

$$
*: V(\mathbb{R}) \longrightarrow V\left({ }^{*} \mathbb{R}\right) .
$$

As usual in the literature, we are making a few blanket assumptions here, namely

1991 Mathematics Subject Classification. 03H05; 03E04; 03C20; 06F30.

Work partially supported by Fondi di Ateneo 2000 grants.

${ }^{1}$ We remark that the notion of hyperreal line in the Internal Set Theory IST of [22] is different. In fact, it is unique because of the axiomatic approach, and its order-properties are of a special nature, due to the strong saturation given by the Idealization axiom. Order-properties of the hyperreal line of BST (a "bounded version" of IST) are studied in [18] (see also [24]). 
- $\mathbb{R}$ and ${ }^{*} \mathbb{R}$ are sets of atoms w.r.t. their respective superstructures, i.e. $x \cap$ $V(\mathbb{R})=\emptyset=y \cap V\left({ }^{*} \mathbb{R}\right)$ for any $x \in \mathbb{R}$ and $y \in{ }^{*} \mathbb{R}$

- $* \mathbb{R}$ is the image of $\mathbb{R}$ under $*$;

- ${ }^{*} x=x$ for all $x \in \mathbb{R}$.

Recall that a bounded elementary extension is a map where the usual elementary extension property is assumed only for those formulae where all quantifiers occur in the bounded forms $\forall x \in y$ or $\exists x \in y$.

An element $x \in V\left({ }^{*} \mathbb{R}\right)$ is called internal if $x \in{ }^{*} y$ for some $y \in V(\mathbb{R})$, or, equivalently, if $x \in \mathcal{I}=\bigcup_{n \in \mathbb{N}}{ }^{*} V_{n}(\mathbb{R})$.

Let $\kappa$ be an infinite cardinal. We say that a nonstandard model is $\kappa$-saturated if every nonempty family $\mathcal{A} \subset \mathcal{I}$ of internal sets of size $|\mathcal{A}|<\kappa$ satisfying the finite intersection property (FIP) has nonempty intersection. Recall that $\mathcal{A}$ has the FIP if, for finitely many $A_{1}, \ldots, A_{n} \in \mathcal{A}$, the intersection $A_{1} \cap \ldots \cap A_{n} \neq \emptyset$.

For more on the foundations and the basics of nonstandard analysis, including the $\kappa$-saturation property, we refer to [1] Section 4.4. For an elementary algebraic approach see [4]. Throughout the paper, by model we always mean a (standard or) nonstandard model of analysis, as defined above.

The paper is organized as follows. In Section 1, we introduce the basic ordertypes involved in the study of the ordering of the hyperreal line, and we state those relations among them which hold in any nonstandard model. This section also contains a short treatment of the connections between these order-types and the main cardinal invariants of the natural group topology of $* \mathbb{R}$. In Section 2 we introduce the technical model-theoretic constructions that will be needed in the sequel. In Section 3 we investigate those relations which depend on the choice of the model. Final remarks and suggestions for further research are contained in Section 4.

The authors are deeply indebted to Furio Honsell, Karel Hrbàček and Renling Jin for many useful discussions and suggestions. The authors are also grateful to the referee for several useful remarks.

\section{THE BASIC ORDER TYPES}

We denote as usual by $\mathbb{N}, \mathbb{R}, \mathbb{R}^{+}$the standard natural, real, and positive real numbers and by $* \mathbb{N},{ }^{*} \mathbb{R},{ }^{*} \mathbb{R}^{+}$the nonstandard (or hyper-) natural, real, and positive real numbers. When considering several models simultaneously, we adopt the usual notation with the name of the model as a superscript. In the study of the ordertheoretic properties of the nonstandard real line, we shall consider order-types of various basic internal and external sets. We list them below, in order to fix our notation:

- $\omega, \lambda$ are the order-types of $\mathbb{N}$ and $\mathbb{R}$, respectively;

- $\rho$ is the order-type of $* \mathbb{R}$;

- $\mu$ is the order-type of $M=\left\{x \in{ }^{*} \mathbb{R}\left|\forall r \in \mathbb{R}^{+}\right| x \mid<r\right\}$ (the monad of 0 );

- $\gamma$ is the order-type of $G=\left\{x \in{ }^{*} \mathbb{R}\left|\exists r \in \mathbb{R}^{+}\right| x \mid<r\right\}$ (the galaxy of 0 ). 
Notice that $G$ and $M$ are convex additive subgroups of ${ }^{*} \mathbb{R}$, hence for any $x \in{ }^{*} \mathbb{R}$ the galaxy of $x$

$$
G_{x}=\left\{t \in{ }^{*} \mathbb{R}\left|\exists r \in \mathbb{R}^{+}\right| t-x \mid<r\right\}=x+G
$$

and the monad of $x$

$$
M_{x}=\left\{t \in{ }^{*} \mathbb{R}\left|\forall r \in \mathbb{R}^{+}\right| t-x \mid<r\right\}=x+M
$$

are order-isomorphic to $G$ and $M$, respectively.

Moreover the quotient groups ${ }^{*} \mathbb{R} / G, G / M,{ }^{*} \mathbb{R} / M$ are ordered groups w.r.t. the induced orderings, since $G$ and $M$ are convex. We say that $x, y \in{ }^{*} \mathbb{R}^{+}$have the same rank if $x / y$ is neither infinite nor infinitesimal. Notice that the (nonstandard) exponential function provides an isomorphism from the ordered additive group $* \mathbb{R} / G$ onto the the ordered multiplicative group of ranks ${ }^{*} \mathbb{R}^{+} /(G \backslash M)^{+}$. In particular, the order-type of the positive part of $* \mathbb{R} / G$ can be seen as coding the ranks (orders) of infinite, and similarly, the negative part of $* \mathbb{R} / G$ can be seen as coding the ranks (orders) of infinitesimal. Therefore we introduce:

- $\theta$ is the order-type of the orders of infinite, or equivalently of $\left({ }^{*} \mathbb{R} / G\right)^{+}$.

We recall some basic facts about order-types that will be used in the sequel without explicit reference.

Let $\sigma$ and $\tau$ be the order-types of $(S,<)$ and $(T,<)$ respectively.

- the sum $\sigma+\tau$ is the order-type of the disjoint union $(S \uplus T,<)$, where the ordering between pairs of elements in $S$ and pairs of elements in $T$ is preserved, and where each element of $S$ precedes all elements of $T$.

- The product $\sigma \tau$ is the order-type of the Cartesian product $(S \times T, \prec)$ with the antilexicographic order, i.e. $(a, b) \prec\left(a^{\prime}, b^{\prime}\right)$ if either $b<b^{\prime}$, or $b=b^{\prime}$ and $a<a^{\prime}$.

- the converse order-type $\sigma^{*}$ of $\sigma$ is the order-type of $(S,>) .^{2}$

We say that $\sigma$ is symmetric if $\sigma^{*}=\sigma$.

- For all order-types $\sigma, \tau$ one has:

$$
\sigma\left(\tau+\tau^{\prime}\right)=\sigma \tau+\sigma \tau^{\prime},(\sigma+\tau)^{*}=\tau^{*}+\sigma^{*}, \text { and }(\sigma \tau)^{*}=\sigma^{*} \tau^{*}
$$

In the following proposition we list some well-known properties and relations among the order-types that we have introduced (see e.g. [30]).

\section{Proposition 1.1.}

(1) The order-types $\lambda, \rho, \gamma, \mu$ are symmetric and dense without endpoints; $\theta$ is dense without endpoints.

(2) $\lambda$ and $\rho$ are the order-types of any open interval in $\mathbb{R}$ and ${ }^{*} \mathbb{R}$, respectively.

(3) The order-type of $G / M$ is $\lambda$, hence $\gamma=\mu \lambda$.

(4) The order-type of $* \mathbb{R} / G$ is $\theta^{*}+1+\theta$, hence $\rho=\gamma\left(\theta^{*}+1+\theta\right)$ and the order-type of $* \mathbb{N}$ is $\omega+\left(\omega^{*}+\omega\right) \theta$.

(5) $\theta$ is the order-type of $\left\{x \in{ }^{*} \mathbb{R}^{+} /(G \backslash M)^{+} \mid x>1\right\}$ (the ranks of infinite).

(6) $\theta^{*}$ is the order-type of $\left\{x \in \mathbb{R}^{+} /(G \backslash M)^{+} \mid x<1\right\}$ (the ranks of infinitesimal).

(7) The order-type of $* \mathbb{R} / M$ is $\lambda\left(\theta^{*}+1+\theta\right)$.

\footnotetext{
${ }^{2}$ This asterisk symbol is not to be confused with the asterisk symbol denoting the nonstandard embedding (the first is placed up right, the latter is placed up left).
} 
(8) $\mu=\gamma \theta+1+\gamma \theta^{*}$.

Let us now turn to the order-types of intervals in $* \mathbb{R} / G$. To this end we introduce:

- $\theta_{x}$ is the order-type of $\left\{G_{y} \in\left({ }^{*} \mathbb{R} / G\right)^{+} \mid G_{y}<G_{x}\right\}$, the initial segment of $\theta$ determined by any infinite $x \in \mathbb{R}^{+}$.

Some basic facts are the following:

\section{Proposition 1.2.}

(1) The order-type $\theta_{x}$ is symmetric for all $x$.

(2) Any open interval $\left(G_{y}, G_{y+x}\right)$ in $* \mathbb{R} / G$ has order-type $\theta_{x}$.

(3) The order-type of any end-interval $\left(G_{x},+\infty\right)$ of $* \mathbb{R} / G$ is $\theta$, hence $\theta=$ $\theta_{x}+1+\theta$.

Proof. The translations and the change-of-sign map are isometries of $* \mathbb{R}$, hence they induce order-isomorphisms on the quotient modulo $G$. Properties 2 and 3 follow by using suitable translations, and Property 1 is obtained by composing the change-of-sign map with a suitable translation.

The problem of the symmetry of the order-type $\theta$ arose since the beginnings of nonstandard analysis, and it has been posed explicitly by E. Zakon in the quoted paper [30], together with the connected question as to whether $\theta=\theta_{x}$ for all (some) $x$. We consider the following possible identities:

(A1) $\theta=\theta^{*}$, i.e. the orders of infinite and of infinitesimal are isomorphic.

(A2) $\forall x\left(\theta=\theta_{x}\right)$, i.e. any initial interval of $\left({ }^{*} \mathbb{R} / G\right)^{+}$is isomorphic to $\left({ }^{*} \mathbb{R} / G\right)^{+}$.

(A3) $\exists x\left(\theta=\theta_{x}\right)$, i.e. some initial interval of $\left({ }^{*} \mathbb{R} / G\right)^{+}$is isomorphic to $\left({ }^{*} \mathbb{R} / G\right)^{+}$.

In this vein, following the analogy with well-known properties of the ordering of the standard and nonstandard real lines, we also consider the identities:

(A4) $\theta=\theta^{*}+1+\theta$, i.e. ${ }^{*} \mathbb{R} / G$ is order-isomorphic to its positive part.

(A5) $\theta=\theta+1+\theta$, i.e. initial intervals and end-intervals of $* \mathbb{R} / G$ are orderisomorphic.

Theorem 1.3. The conditions $(A 1),(A 2),(A 3),(A 4)$, and $(A 5)$ are equivalent in any nonstandard model.

Proof. The implication $(A 2) \Rightarrow(A 3)$ is trivial. The implications $(A 3) \Rightarrow(A 1),(A 3) \Rightarrow(A 4)$, and $(A 3) \Rightarrow(A 5)$ are immediate consequences of the symmetry of $\theta_{x}$ and of the identity $\theta=\theta_{x}+1+\theta$ of Proposition 1.2.

Condition $(A 5)$ means essentially that there is an initial segment of $\theta$ which is order-isomorphic to $\theta$, hence $(A 3)$. Since $(A 4)$ yields immediately the symmetry of $\theta$, we are left to show that $(A 1) \Rightarrow(A 2)$. So, assume $(A 1)$. Then, for any $x$,

$$
\theta=\theta^{*}=\left(\theta_{x}+1+\theta\right)^{*}=\theta^{*}+1+\theta_{x}^{*}=\theta+1+\theta_{x} .
$$

Hence $\theta_{x}$ is an open end-interval of $\theta$, which is of type $\theta$ by Proposition 1.2, and $(A 2)$ follows. 
In the cited paper [30], the question is raised as to whether the monads are orderisomorphic to the whole nonstandard real line. In order to better investigate this question, we introduce the following identities, which parallel $(A 1)-(A 5)$ above.

(B1) $\mu=\rho$, i.e. the monad is isomorphic to the whole line.

(B2) $\mu=\mu+1+\mu$, i.e. the monad is isomorphic both to its positive and its negative part.

(B3) $\mu=\gamma \theta$, i.e. the monad is isomorphic to the positive infinites.

(B4) $\rho=\gamma \theta$, i.e. the hyperreal line is isomorphic to the positive infinites.

(B5) $\gamma \theta=\gamma \theta^{*}$, i.e. the positive and the negative infinites are isomorphic.

Theorem 1.4. The conditions $(B 1),(B 2),(B 3),(B 4)$, and (B5) are equivalent in any nonstandard model.

Proof. The implications $(B 1) \Rightarrow(B 2)$ and $(B 3) \Rightarrow(B 5)$ are immediate.

$(B 2) \Rightarrow(B 3)$ follows from point 8 of Proposition 1.1, because every initial interval of $\mu$ has order-type $\gamma \theta$.

Moreover, by the same point, (B4) implies $\mu=\gamma \theta+1+\gamma \theta^{*}=\rho+1+\rho^{*}=\rho$, that is $(B 1)$.

Finally, every open end-interval of $\gamma \theta$ has order-type $\rho$. So, if $\gamma \theta$ is symmetric, then also its initial intervals have order-type $\rho$, hence $\gamma \theta=\rho+1+\rho=\rho$, and $(B 5) \Rightarrow(B 4)$ follows.

As a straightforward consequence of the above theorems, we get the

Corollary 1.5. The following implications hold in any nonstandard model:

(1) $\theta=\theta^{*} \Longrightarrow \mu=\rho=\gamma \theta$.

(2) $\mu=\rho \Longrightarrow \operatorname{cof} \theta=\operatorname{cof} \theta^{*}$.

In Section 3, we shall show that neither of the above implications can be reversed, in general. Since cofinalities have particular relevance for our investigation, we state here the following straightforward identities:

Remark 1.6.

(1) $\operatorname{cof} \lambda=\operatorname{cof} \gamma=\omega$.

(2) $\operatorname{cof} \rho=\operatorname{cof} * \mathbb{N}=\operatorname{cof} \theta$.

(3) $\operatorname{cof} \mu=\operatorname{cof} \theta^{*}=\operatorname{cof} \theta_{x}$.

We conclude this section with some interesting topological properties of the hyperreal line. The hyperreal line $* \mathbb{R}$ carries a natural order topology which coincides with the natural group topology having the convex subgroups as a neighborhood basis of 0 . This topology on ${ }^{*} \mathbb{R}$ is usually called the $Q$-topology. A detailed study of the $Q$-topology lies outside the scope of this paper (see, e.g. [25, 30]). We restrict ourselves here to consider its main cardinal invariants, as introduced in [5]. We shall see that all of them are explicitly computable in terms of the order-type $\theta$.

Let $X$ be a topological space of cardinality k. We consider the following topological invariants of $X$ : 
$\chi$ : the character, i.e. the least cardinal $\kappa$ s.t. any point has neighborhood basis of size $\kappa$.

$\psi$ : the pseudocharacter, i.e. the least cardinal $\kappa$ s.t. any point is the intersection of a family of neighborhoods of size $\kappa$.

$\tau$ : the tightness, i.e. the least cardinal $\kappa$ s.t. for any set $C$ and any point $\mathbf{x} \in \bar{C}$, $x$ lies in the closure of some subset of $C$ of size $\kappa$.

$\Delta$ : the diagonal weight, i.e. the least size of a family of open subsets of $X^{2}$ whose intersection is the diagonal.

d : the density, i.e. the least size of a dense subset.

s : the spread, i.e. the supremum of the sizes of discrete subsets.

e : the extent, i.e. the supremum of the sizes of discrete closed subsets.

1 : the Lindelof number, i.e. the least cardinal $\kappa$ s.t. any open cover has a subcover of size $\kappa$.

c : the cellularity, i.e. the supremum of the sizes of disjoint families of open sets.

$\mathbf{w}$ : the weight, i.e. the least size of an open basis.

$\mathbf{n}$ : the net weight, i.e. the least size of a family generating all open sets.

$\mathbf{u}$ : (only for Tychonoff spaces) the uniform weight, i.e. the least size of a uniformity basis inducing the topology of $X$.

In many cases also the corresponding hereditary invariants are of interest. Thus, if $\mathbf{x}$ is any of the above cardinals, then $\mathbf{h x}$ denotes the "hereditary $\mathbf{x}$ ", i.e.

$$
\mathbf{h} \mathbf{x}(X)=\sup \{\mathbf{x}(Y) \mid Y \subseteq X\}
$$

We are now ready to compute all these cardinals in terms of $\theta$, namely

Theorem 1.7. The following equalities hold in any nonstandard model:

(1) $\chi=\psi=\tau=\mathbf{u}=\Delta=\operatorname{cof} \theta$.

(2) $\mathbf{w}=\mathbf{n}=\mathbf{d}=\mathbf{h d}=\mathbf{s}=\mathbf{h s}=\mathbf{l}=\mathbf{h l}=\mathbf{c}=\mathbf{h} \mathbf{c}=\mathbf{e}=\mathbf{h e}=|\theta|$.

(3) $|\theta| \leq \mathbf{k} \leq|\theta|^{\operatorname{cof} \theta}$.

Proof. In any topological space one has $\mathbf{h c}=\mathbf{h e}=\mathbf{h} \mathbf{s}=\mathbf{s}$ (see [5]). Moreover the following inequalities are stated in Problem 3.12.4 of [5] as holding in any linearly ordered topological space:

$$
\mathbf{w}=\mathbf{n} \geq \mathbf{d}=\mathbf{h d} \geq \mathbf{c}=\mathbf{h l}=\mathbf{h} \mathbf{c} \geq \mathbf{l} \geq \mathbf{e} \quad \text { and } \quad \chi=\psi=\tau .
$$

Now $|\theta|$ is both the cardinality of the hypernatural and of the hyperrational numbers. Therefore $|\theta| \geq \mathbf{w}$ since the open intervals with hyperrational endpoints are a basis of the order-topology of ${ }^{*} \mathbb{R}$. Moreover $\mathbf{e} \geq|\theta|$ since ${ }^{*} \mathbb{N}$ is a closed discrete subspace of $* \mathbb{R}$. All equalities of Point 2 are thus proved.

In order to get Point 1 , observe first that the hypermetric entourages $U_{\epsilon}=$ $\{(x, y)|| x-y \mid<\epsilon\}$, as $\epsilon$ runs over a coinitial subset of $* \mathbb{R}^{+}$, are a nested uniformity basis inducing the group topology of $* \mathbb{R}$. Hence ${ }^{*} \mathbb{R}$ is a $\kappa$-metric space in the sense of [10] (with $\kappa=\operatorname{cof} \theta$ ), and so $\mathbf{u} \leq \operatorname{cof} \theta$. 
Moreover no family of neighborhoods of a point $x$ can have intersection $\{x\}$ if its size is less than $\operatorname{cof} \theta$. It follows that $\Delta \geq \psi \geq \operatorname{cof} \theta$. Combining these inequalities with $\Delta \leq \mathbf{u}$, which holds in any uniform space, one gets all of Point 1 .

Finally, the first inequality of Point 3 is obvious, and the second one follows from the fact that any point of $* \mathbb{R}$ is the intersection of a suitable $\kappa$-sequence of open intervals with hyperrational endpoints.

\section{Constructing models of nOnstandard AnAlysis}

In this section we introduce the technical machinery that will be used in the next section. All our constructions of models will be obtained by means of suitable iterations of two special kinds of elementary extensions, namely ultrapowers and internal ultrapowers.

We assume that the reader is familiar with the ultrapower construction (see e.g. [1] Section 4.1). If $D$ is an ultrafilter over the cardinal $\kappa$, we denote by $\mathcal{M}_{D}^{\kappa}$ the ultrapower of $\mathcal{M}$ modulo $D$.

We shall also consider the more general notion of limit ultrapower.

Definition 2.1. Let $F$ be a filter of equivalences over $\kappa$. The limit ultrapower $\mathcal{M}_{D}^{\kappa} \mid F$ is the submodel of $\mathcal{M}_{D}^{\kappa}$ whose universe consists of the $D$-equivalence classes $f_{D}$ of functions $f: \kappa \rightarrow \mathcal{M}$ which are "compatible with $F$ ", i.e. such that

$$
\{(i, j) \in \kappa \times \kappa \mid f(i)=f(j)\} \in F .
$$

The diagonal embedding $d: \mathcal{M} \rightarrow \mathcal{M}_{D}^{\kappa} \mid F$ is still defined, and it satisfies Los theorem (see e.g. [1] Section 6.4).

The notion of limit ultrapower is crucial in our context because of H.J. Keisler's characterization theorem:

Theorem 2.2 (see $[20])$. The map $*: V(\mathbb{R}) \rightarrow V(* \mathbb{R})$ is a nonstandard embedding if and only if there exist

- an ultrafilter $D$ over some cardinal $\kappa$;

- a filter $F$ of equivalences over $\kappa$;

- an isomorphism $\pi$ of the bounded limit ultrapower $\bigcup_{n \in \mathbb{N}} V_{n}(\mathbb{R})^{\kappa}{ }_{D} \mid F$ onto the internal submodel $\mathcal{I} \subset V\left({ }^{*} \mathbb{R}\right)$

such that the following diagram commutes:

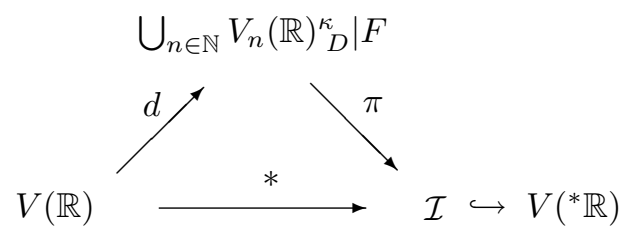

In the sequel we shall directly identify nonstandard models with limit ultrapowers of $V(\mathbb{R})$. This can be done without loss of generality. In fact, on the one hand, every nonstandard embedding is (up to isomorphism) the diagonal embedding of the standard model into a bounded limit ultrapower by the above theorem. On the other hand, given a limit ultrapower $V(\mathbb{R})_{D}^{\kappa} \mid F$, the corresponding nonstandard embedding is the composition $*=\pi \circ d: V(\mathbb{R}) \rightarrow V\left({ }^{*} \mathbb{R}\right)$, where

- $d: V(\mathbb{R}) \rightarrow \bigcup_{n \in \mathbb{N}} V_{n}(\mathbb{R})^{\kappa}{ }_{D} \mid F$ is the diagonal embedding; 
- $\pi: \bigcup_{n \in \mathbb{N}} V_{n}(\mathbb{R})_{D}^{\kappa} \mid F \rightarrow T$ is a Mostowski collapse such that - $\pi$ induces a bijection of $\mathbb{R}_{D}^{\kappa} \mid F$ onto ${ }^{*} \mathbb{R}$,

$-{ }^{*} \mathbb{R}$ is a set of atoms w.r.t. its superstructure,

$-\pi(d(r))=r$ for every $r \in \mathbb{R}$.

Notice that $T \subset V\left({ }^{*} \mathbb{R}\right)$ is transitive up to elements in ${ }^{*} \mathbb{R}$, i.e. $t \subset T$ for all $t \in T$ with $t \notin * \mathbb{R}$.

Definition 2.3. Let $\mathcal{M}=V(\mathbb{R})_{E}^{\nu} \mid F$ be a nonstandard model, and let $D$ be an ultrafilter over a cardinal $\kappa$. The internal ultrapower of $\mathcal{M}$ modulo $D$ is the model

$$
\left[\mathcal{M}_{D}^{\kappa}\right]=\left(V(\mathbb{R})_{D}^{\kappa}\right)_{E}^{\nu} \mid F \text {. }
$$

The internal embedding

$$
e: \mathcal{M} \longrightarrow\left[\mathcal{M}_{D}^{\kappa}\right]
$$

maps the $E$-equivalence class $f_{E}$ of any function $f: \nu \rightarrow V(\mathbb{R})$ (compatible with $F)$ to the class $\varphi_{E}$ of the function $\varphi=d \circ f$, where $d: V(\mathbb{R}) \rightarrow V(\mathbb{R})^{\kappa}{ }_{D}$ is the diagonal embedding.

It is immediate to verify that $e$ is an elementary embedding. When no ambiguity can arise, we identify every nonstandard real $x \in{ }^{*} \mathbb{R}^{\mathcal{M}}$ with its diagonal image $d(x) \in{ }^{*} \mathbb{R}^{\mathcal{U}}$ in the ultrapower $\mathcal{U}=\mathcal{M}_{D}^{\kappa}$, so as to have ${ }^{*} \mathbb{R}^{\mathcal{M}} \subset{ }^{*} \mathbb{R}^{\mathcal{U}}$. Similarly, we identify each $x \in{ }^{*} \mathbb{R}^{\mathcal{M}}$ with $e(x) \in{ }^{*} \mathbb{R}^{\mathcal{V}}$ in the internal ultrapower $\mathcal{V}=\left[\mathcal{M}_{D}^{\kappa}\right]$.

In the next lemma we itemize a few basic properties that will be useful in the sequel. A detailed study of this kind of extentions can be found in [23].

Lemma 2.4. Let $D$ be a countably incomplete ultrafilter over a cardinal $\kappa$ and let $\mathcal{M}$ be a model. Let $\mathcal{U}=\mathcal{M}_{D}^{\kappa}$ be the ultrapower of $\mathcal{M}$ modulo $D$ and let $\mathcal{V}=\left[\mathcal{M}^{\kappa}{ }_{D}\right]$ be the corresponding internal ultrapower.

(1) If $\operatorname{cof} \theta^{\mathcal{M}}>\kappa$ then $* \mathbb{N}^{\mathcal{M}}$ is cofinal in ${ }^{*} \mathbb{N}^{\mathcal{U}}$, hence $\operatorname{cof} \theta^{\mathcal{M}}=\operatorname{cof} \theta^{\mathcal{U}}$.

(2) Let $S$ be an initial cut of ${ }^{*} \mathbb{N}^{\mathcal{M}}\left(\right.$ or $\left.{ }^{*} \mathbb{R}^{\mathcal{M}}\right)$. Then $S_{D}^{\kappa}$ is an initial cut of ${ }^{*} \mathbb{N}^{\mathcal{U}}$ (or ${ }^{*} \mathbb{R}^{\mathcal{U}}$, respectively).

(3) $\theta^{\mathcal{M}}$ is bounded from below in $\theta^{\mathcal{U}}$, hence there are elements in the monad $x \in M^{\mathcal{U}}$ such that $x>x^{\prime}$ for all $x^{\prime} \in M^{\mathcal{M}}$.

(4) There are elements $x \in \theta^{\mathcal{U}}$ such that $\left|\theta_{x}^{\mathcal{U}}\right| \leq 2^{\kappa}$, hence $\operatorname{cof} \theta^{* \mathcal{U}} \leq 2^{\kappa}$.

(5) $* \mathbb{N}^{\mathcal{M}}$ is an initial cut of ${ }^{*} \mathbb{N}^{\mathcal{V}}$, hence $\operatorname{cof} \theta^{* \mathcal{M}}=\operatorname{cof} \theta^{* \mathcal{V}}$, and the monad $M^{\mathcal{M}}$ is cofinal in $M^{\mathcal{V}}$.

In order to get models with the desired structural properties, we concentrate on iterated ultrapowers and iterated internal ultrapowers given by regular ultrafilters.

Let $\mathcal{G}$ be any fixed (standard or nonstandard) model. Let $\delta$ be a limit ordinal and let $\mathcal{D}=\left\langle\left(D_{\alpha}, \kappa_{\alpha}\right)\right\rangle_{\alpha<\delta}$ be a $\delta$-sequence where every $D_{\alpha}$ is a regular ultrafilter over the cardinal $\kappa_{\alpha}$.

Definition 2.5. The models $\mathcal{U}(\mathcal{G}, \delta, \mathcal{D})$ and $\mathcal{V}(\mathcal{G}, \delta, \mathcal{D})$ are the direct limits of the systems $\left\langle\mathcal{U}_{\alpha} ; d_{\alpha}\right\rangle_{\alpha<\delta}$ and respectively $\left\langle\mathcal{V}_{\alpha} ; e_{\alpha}\right\rangle_{\alpha<\delta}$, where:

- $\mathcal{U}_{0}=\mathcal{V}_{0}=\mathcal{G}$;

- $\mathcal{U}_{\alpha+1}=\mathcal{U}_{\alpha} \stackrel{\kappa_{\alpha}}{D_{\alpha}}$ is the ultrapower of $\mathcal{U}_{\alpha}$ modulo $D_{\alpha}$.

- $d_{\alpha}: \mathcal{U}_{\alpha} \rightarrow \mathcal{U}_{\alpha+1}$ is the diagonal embedding. 
- $\mathcal{V}_{\alpha+1}=\left[\mathcal{V}_{\alpha} \begin{array}{c}\kappa_{\alpha} \\ D_{\alpha}\end{array}\right]$ is the internal ultrapower of $\mathcal{V}_{\alpha}$ modulo $D_{\alpha}$.

- $e_{\alpha}: \mathcal{V}_{\alpha} \rightarrow \mathcal{V}_{\alpha+1}$ is the internal embedding.

- For limit $\beta<\delta, \mathcal{U}_{\beta}$ and $\mathcal{V}_{\beta}$ are the direct limits of $\left\langle\mathcal{U}_{\alpha} ; d_{\alpha}\right\rangle_{\alpha<\beta}$ and $\left\langle\mathcal{V}_{\alpha} ; e_{\alpha}\right\rangle_{\alpha<\beta}$, respectively.

Notice that the models obtained at each limit step are isomorphic to limit ultrapowers, hence the internal ultrapower is well-defined. We summarize in the following lemmata some basic properties of these models. The proofs require only straightforward applications of Lemma 2.4 and Definition 2.5.

Lemma 2.6. Let $\mathcal{U}=\mathcal{U}(\mathcal{G}, \delta, \mathcal{D})$ be the $\delta$-iterated ultrapower of $\mathcal{G}$ modulo $\mathcal{D}=$ $\left\langle\left(D_{\alpha}, \kappa_{\alpha}\right)\right\rangle_{\alpha<\delta}$.

(1) $\operatorname{cof} \theta^{* \mathcal{U}}=\operatorname{cof} \delta$.

(2) If $\operatorname{cof} \theta^{\mathcal{G}}>\kappa_{\alpha}$ for all $\alpha$, then ${ }^{*} \mathbb{N}^{\mathcal{G}}$ is cofinal in $* \mathbb{N}^{\mathcal{U}}$, hence $\operatorname{cof} \theta^{\mathcal{U}}=\operatorname{cof} \theta^{\mathcal{G}}$.

(3) If $\kappa_{\alpha} \geq \operatorname{cof} \theta^{\mathcal{U}_{\alpha}}$ for all $\alpha$, then $\operatorname{cof} \theta^{\mathcal{U}}=\operatorname{cof} \delta$. In particular this holds when $\kappa_{\alpha}=\beth_{\alpha}(\nu),{ }^{3}$ with $\nu \geq \operatorname{cof} \theta^{\mathcal{G}}$.

(4) Let $\nu \leq \operatorname{cof} \delta$. If $\left\{\alpha \mid \mathcal{U}_{\alpha}\right.$ is $\nu$-saturated $\}$ is cofinal in $\delta$, then $\mathcal{U}$ is $\nu$ saturated.

In the next section we shall use good ultrafilters in order to obtain saturated models. For the definition we refer to [1], where also the following fundamental facts are proved:

- Every ultrapower modulo a countably incomplete $\nu$-good ultrafilter is $\nu$ saturated (provided the size of the language is not larger than $\nu$ ).

- Over any cardinal $\kappa$ there exist $\kappa^{+}$-good countably incomplete ultrafilters.

Lemma 2.7. Let $\mathcal{V}=\mathcal{V}(\mathcal{G}, \delta, \mathcal{D})$ be the $\delta$-iterated internal ultrapower of $\mathcal{G}$ modulo $\mathcal{D}=\left\langle\left(D_{\alpha}, \kappa_{\alpha}\right)\right\rangle_{\alpha<\delta}$. Then

(1) $\operatorname{cof} \theta^{\mathcal{V}}=\operatorname{cof} \delta$.

$(2) * \mathbb{N}^{\mathcal{V}}$ is an initial segment of $* \mathbb{N}^{\mathcal{V}}$ for all $\alpha<\delta$, hence $\theta_{x}^{\mathcal{V}}=\theta_{x}{ }^{\mathcal{V}}$ for all $x \in \mathcal{V}_{\alpha}$. In particular $\operatorname{cof} \theta^{* \mathcal{V}}=\operatorname{cof} \theta^{* \mathcal{V}_{\alpha}}=\operatorname{cof} \theta^{* \mathcal{G}}$ for all $\alpha<\delta$.

\section{Model-SEnsitive ORDER-TheORETIC PROPERTIES}

In this section we study order-theoretic properties which depend on the choice of the model. To this aim we use the construction techniques introduced above.

First of all we build symmetric models of arbitrary cofinality.

Theorem 3.1. Let $\kappa$ be a regular cardinal. There exist $\kappa$-saturated models where $\theta=\rho$ and $\operatorname{cof} \theta=\kappa$. In particular $\theta=\theta^{*}$ is compatible with arbitrary values of $\operatorname{cof} \theta$.

Proof. Let $\mathcal{G}$ be a model and put $\kappa_{\alpha}=\beth_{\alpha}(|\mathcal{G}|)$. Let $\mathcal{U}=\mathcal{U}(\mathcal{G}, \kappa, \mathcal{D})$ be the $\kappa$ iterated ultrapower of $\mathcal{G}$ modulo $\mathcal{D}=\left\langle\left(D_{\alpha}, \kappa_{\alpha}\right)\right\rangle_{\alpha<\kappa}$, where $D_{\alpha}$ is a countably incomplete $\kappa_{\alpha}^{+}$-good ultrafilter over $\kappa_{\alpha}$. Then $\operatorname{cof} \theta=\operatorname{cof} \theta^{*}=\kappa$ and $\mathcal{U}$ is $\kappa$ saturated by Lemma 2.6 .

\footnotetext{
${ }^{3}$ Recall that $\beth_{\alpha}(\nu)$ is inductively defined by $\beth_{0}(\nu)=\nu, \beth_{\alpha+1}(\nu)=2^{\beth_{\alpha}(\nu)}$ and, for limit $\beta$, $\beth_{\beta}(\nu)=\sup \left\{\beth_{\alpha}(\nu) \mid \alpha<\beta\right\}$.
} 
We are left to prove that $\theta=\rho$. We want to show that there exists an orderisomorphism $\Psi: X \rightarrow{ }^{*} \mathbb{R}$, where $X \subset{ }^{*} \mathbb{R}$ is a set of representatives for $\theta$ (i.e. $G_{x} \neq G_{y}$ for all $x \neq y$ in $\mathrm{X}$, and $\left.\theta=\left\{G_{x} \mid x \in X\right\}\right)$.

For each $\alpha<\kappa$, put ${ }^{*} \mathbb{R}_{\alpha}={ }^{*} \mathbb{R}^{\mathcal{U}_{\alpha}}$. By transfinite induction, we define:

- $\operatorname{sets} X_{\alpha} \subset{ }^{*} \mathbb{R}_{\alpha}$ for even $\alpha$,

- order-preserving maps $f_{\alpha}: X_{\alpha} \rightarrow{ }^{*} \mathbb{R}_{\alpha+1}$ for even $\alpha$, and

- order-preserving maps $g_{\alpha}:{ }^{*} \mathbb{R}_{\alpha} \rightarrow X_{\alpha+1}$ for odd $\alpha$

in such a way that the following properties are satisfied:

(1) $X_{\alpha}$ is a set of representatives for $\theta^{\mathcal{U}_{\alpha}}$;

(2) $g_{\alpha+1}\left(f_{\alpha}(b)\right)=b$ for all $b \in X_{\alpha}$ ( $\alpha$ even);

(3) $f_{\alpha+1}\left(g_{\alpha}(a)\right)=a$ for all $a \in{ }^{*} \mathbb{R}_{\alpha}(\alpha$ odd $)$.

If $\alpha=0$, pick $X_{0}$ a set of representative for $\theta^{\mathcal{U}_{0}}$ and let $f_{0}: X_{0} \rightarrow{ }^{*} \mathbb{R}_{1}$ be the identity map.

Now assume $\alpha+1$ is odd. Let $\mathbb{F}\left({ }^{*} \mathbb{R}\right)$ be the (internal) collection of all internal order-preserving partial functions from ${ }^{*} \mathbb{R}$ into itself, and, for fixed $a, b \in{ }^{*} \mathbb{R}$ and $n \in \mathbb{N}$, put:

- $\Lambda(a)=\left\{g \in \mathbb{F}\left({ }^{*} \mathbb{R}\right) \mid f_{\alpha}(a) \in \operatorname{dom} g \Rightarrow g\left(f_{\alpha}(a)\right)=a\right\}$

- $\Gamma(b)=\left\{g \in \mathbb{F}\left({ }^{*} \mathbb{R}\right) \mid b \in \operatorname{dom} g\right\}$;

- $\Delta(n)=\left\{g \in \mathbb{F}\left({ }^{*} \mathbb{R}\right) \mid \forall x, y \in \operatorname{dom} g(x \neq y \Rightarrow|g(x)-g(y)|>n)\right\}$.

Since both $* \mathbb{R}$ and $* \mathbb{R} / G$ are dense without endpoints, the family

$$
\mathcal{F}=\left\{\Lambda(a) \mid a \in X_{\alpha}\right\} \cup\left\{\Gamma(b) \mid b \in{ }^{*} \mathbb{R}_{\alpha+1}\right\} \cup\{\Delta(n) \mid n \in \mathbb{N}\}
$$

satisfies the FIP. Moreover $|\mathcal{F}| \leq\left|\mathcal{U}_{\alpha+1}\right|=\kappa_{\alpha+1}$, hence, by $\kappa_{\alpha+1}^{+}$-saturation, there is a function $g$ in $\mathcal{U}_{\alpha+2}$ s.t. $g \in \bigcap \mathcal{F}$. Since $g$ is defined for all $b \in{ }^{*} \mathbb{R}_{\alpha+1}$, we can take as $g_{\alpha+1}$ the restriction $g \uparrow^{*} \mathbb{R}_{\alpha+1}$. Such a $g_{\alpha+1}$ satisfies the desired property.

Now, assume that $\alpha+2$ is even. First, pick $X_{\alpha+2} \supset X_{\alpha}$ a set of representatives for $\theta^{\mathcal{U}_{\alpha+2}}$, and put

$$
\Lambda^{\prime}(a)=\left\{f \in \mathbb{F}\left({ }^{*} \mathbb{R}\right) \mid g_{\alpha+1}(a) \in \operatorname{dom} f \Rightarrow f\left(g_{\alpha+1}(a)\right)=a\right\}
$$

and

$$
\mathcal{F}^{\prime}=\left\{\Lambda^{\prime}(a) \mid a \in{ }^{*} \mathbb{R}_{\alpha+1}\right\} \cup\left\{\Gamma(b) \mid b \in X_{\alpha+2}\right\}
$$

Proceeding as above we can see that the family $\mathcal{F}^{\prime}$ has the FIP. Since $\left|\mathcal{F}^{\prime}\right| \leq$ $\left|\mathcal{U}_{\alpha+2}\right|=\kappa_{\alpha+2}$, by $\kappa_{\alpha+2}^{+}$-saturation we can pick a function $f$ in $\mathcal{U}_{\alpha+3}$ s.t. $f \in \bigcap \mathcal{F}^{\prime}$. Then dom $f$ includes $X_{\alpha+2}$, and $f_{\alpha+2}$ can be defined as the restriction of $f$ to $X_{\alpha+2}$, thus satisfying the required conditions.

If $\beta$ is a limit (hence even), let

$$
X_{\beta}=\bigcup\left\{X_{\alpha} \mid \alpha \text { even }<\beta\right\} \text { and } f_{\beta}=\bigcup\left\{f_{\alpha} \mid \alpha \text { even }<\beta\right\} .
$$

By induction hypothesis $X_{\beta}$ is a set of representatives for $\theta^{\mathcal{U}_{\beta}}$, and the functions $f_{\alpha}$ are coherent, hence $f_{\beta}$ satisfies the desired properties. Clearly, conditions 1-3 above imply that for each limit $\beta$ the function $f_{\beta}$ is an order-isomorphism of $X_{\beta}$ onto ${ }^{*} \mathbb{R}_{\beta}$, whose inverse is $\bigcup\left\{g_{\alpha} \mid \alpha\right.$ odd $\left.<\beta\right\}$.

It follows at once that $X=\bigcup\left\{X_{\alpha} \mid \alpha\right.$ even $\left.<\kappa\right\}$ is a set of representatives for $\theta$ and that $\Psi=\bigcup\left\{f_{\alpha} \mid \alpha\right.$ even $\left.<\kappa\right\}$ is an order-isomorphism from $X$ onto ${ }^{*} \mathbb{R}$, whose inverse is $\Psi^{-1}=\bigcup\left\{g_{\alpha} \mid \alpha\right.$ odd $\left.<\kappa\right\}$.

Next we show that coinitiality and cofinality of $\theta$ can be arbitrarily chosen. 
Theorem 3.2. Let $\kappa_{1}$ and $\kappa_{2}$ be regular cardinals, and put $\kappa=\min \left\{\kappa_{1}, \kappa_{2}\right\}$. There exist $\kappa$-saturated models such that:

(i) $\operatorname{cof} \theta^{*}=\kappa_{1}$ and $\operatorname{cof} \theta=\kappa_{2}$.

Moreover, if $\xi, \eta$ are cardinals such that $\eta>\xi \geq \kappa_{1}$, then the models can be chosen so as to satisfy

(ii) $\left|\theta_{x}\right|=\xi^{<\kappa_{1}}$ and $\left|\theta_{y}\right|=\eta^{<\kappa_{1}}$ for suitable $x, y$. ( $\xi^{\omega}$ and respectively $\eta^{\omega}$ if $\left.\kappa_{1}=\omega\right)$.

Proof. Assume first that $\kappa=\zeta^{+}$is a successor cardinal. Take $\mathcal{G}$ a ground model where cof $\theta=\kappa_{2}$. Consider $\mathcal{U}=\mathcal{U}(\mathcal{G}, \delta, \mathcal{D})$ where cof $\delta=\kappa_{1}$ and $\mathcal{D}$ is constantly equal to $(D, \zeta), D$ a fixed $\zeta^{+}$-good ultrafilter over $\zeta$. Then $\mathcal{U}$ satisfies the conditions required by Lemma 2.6 , hence it is $\kappa$ saturated and satisfies (i).

In order to satisfy condition (ii), it suffices to choose $\delta=\eta+\xi+\kappa_{1}$ (ordinal sum). Simply pick a positive infinite $x$ in $\mathcal{U}_{\eta+1}$ preceding all those in $\mathcal{U}_{\eta}$ and an infinite $y \in{ }^{*} \mathbb{R}^{\mathcal{U}_{1}}$ preceding all those in $\mathcal{G}$. Then

$$
\left|\theta_{x}\right|=\sup \left\{|\alpha|^{\zeta} \mid \eta+\alpha<\delta\right\}=\xi^{\zeta} \text { and }\left|\theta_{y}\right|=\sup \left\{|\alpha|^{\zeta} \mid \alpha<\delta\right\}=\eta^{\zeta} .
$$

Let us now assume that $\kappa=\bigcup_{\alpha<\kappa} \zeta_{\alpha}$ is a regular uncountable limit cardinal (i.e. weakly inaccessible). We slightly modify the preceding construction. Take $\mathcal{G}$ a ground model where $\operatorname{cof} \theta=\kappa_{2}$. Let $\delta$ be a limit ordinal with $\operatorname{cof} \delta=\kappa_{1}$ and let $\varphi: \delta \rightarrow \kappa$ be a function such that $\varphi^{-1}(\alpha)$ is cofinal in $\delta$ for all $\alpha<\kappa$. For $\alpha<\kappa$ let $D_{\alpha}$ be a $\zeta_{\alpha}^{+}$-good ultrafilter over $\zeta_{\alpha}$ and put $\mathcal{D}=\left\langle\left(D_{\varphi(\beta)}, \zeta_{\varphi(\beta)}\right)\right\rangle_{\beta<\delta}$.

Then $\mathcal{U}=\mathcal{U}(\mathcal{G}, \delta, \mathcal{D})$ is $\kappa$-saturated and satisfies conditions (i) by Lemma 2.6. Condition (ii) can be met by taking $\mathcal{G}$ and $\delta$ exactly as in the successor case.

We are left with $\kappa=\omega$. We do not need to care about saturation, since every nonstandard model is $\aleph_{0}$-saturated.

Suppose first that $\kappa_{2}>\kappa_{1}=\omega$. Then we can take $\zeta=\omega$ and proceed exactly as in the successor case.

Finally suppose that $\kappa_{1} \geq \kappa_{2}=\omega$. Then we can start with a model $\mathcal{G}$ where $\operatorname{cof} \theta^{*}=\kappa_{1}$ and property (ii) is satisfied. In order to adjust the cofinality of $\theta$, we take an iteration of $\omega$ internal ultrapowers $\mathcal{V}=\mathcal{V}(\mathcal{G}, \omega, \mathcal{D})$ where $\mathcal{D}$ is any sequecence. In this way, by Lemma 2.7, we preserve the initial part of $\theta$ and get $\operatorname{cof} \theta=\omega$.

Remark that $\kappa$-saturation is best possible in the above theorem, because neither $\operatorname{cof} \theta$ nor $\operatorname{cof} \theta^{*}$ can be less than $\kappa$ in a $\kappa$-saturated model. The goal of the constructions above was precisely that of getting the maximal saturation with prescribed cofinalities. Giving up with saturation, we could have chosen any model of the form $\mathcal{V}\left(\mathcal{U}\left(\mathcal{G}, \delta_{1}, \mathcal{D}_{1}\right), \delta_{2}, \mathcal{D}_{2}\right)$ with $\operatorname{cof} \delta_{i}=\kappa_{i}$.

The importance of $\kappa$-saturation in our context is witnessed by the following lemma, which provides a useful sufficient condition for the identity $\mu=\rho$.

Lemma 3.3. Assume that $\operatorname{cof} \theta=\operatorname{cof} \theta^{*}=\kappa$. If the model is $\kappa$-saturated, then $\mu=\rho$.

Proof. It is enough to show that there is an order-isomorphism between the set of positive infinitesimals $M^{+}$and ${ }^{*} \mathbb{R}^{+}$. Let $\left\{a_{\alpha} \mid \alpha<\kappa\right\} \subset M^{+}$and $\left\{b_{\alpha} \mid \alpha<\kappa\right\} \subset$ ${ }^{*} \mathbb{R}^{+}$be increasing cofinal $\kappa$-sequences. By transfinite induction, we define a family $\left\{f_{\alpha} \mid \alpha<\kappa\right\}$ of internal functions such that for every $\alpha$ : 
(1) $f_{\alpha}:\left(0, a_{\alpha}\right] \rightarrow\left(0, b_{\alpha}\right]$ is an order-isomorphism.

(2) $f_{\alpha}$ extends $f_{\beta}$ for all $\beta<\alpha$.

Pick $f_{0}$ an internal order-isomorphism between the intervals $\left(0, a_{0}\right]$ and $\left(0, b_{0}\right]$ $\left(\right.$ say $\left.f_{0}(x)=x \cdot \frac{b_{0}}{a_{0}}\right)$.

At the inductive step $\alpha>0$, proceed as follows. For each $\beta<\alpha$, let

$F_{\beta}=\left\{f:\left(0, a_{\alpha}\right] \rightarrow\left(0, b_{\alpha}\right] \mid f\right.$ is an internal order-isomorphism extending $\left.f_{\beta}\right\}$.

Notice that all $F_{\beta}$ are internal and nonempty. For instance, the function $\varphi_{\beta}$

$$
\varphi_{\beta}(x)=\left\{\begin{array}{cl}
f_{\beta}(x) & \text { if } x \in\left(0, a_{\beta}\right] \\
\left(x-a_{\beta}\right) \frac{b_{\alpha}-b_{\beta}}{a_{\alpha}-a_{\beta}}+b_{\beta} & \text { if } x \in\left(a_{\beta}, a_{\alpha}\right]
\end{array}\right.
$$

belongs to $F_{\beta}$.

Notice also that $F_{\beta} \subseteq F_{\beta^{\prime}}$ for $\beta^{\prime}<\beta<\alpha$, because $f_{\beta}$ extends $f_{\beta^{\prime}}$ by the inductive hypothesis. In particular, $F_{\beta_{1}} \cap \ldots \cap F_{\beta_{n}}=F_{\max }\left\{\beta_{1}, \ldots, \beta_{n}\right\}$, and the FIP holds. Moreover, since $\left|\left\{F_{\beta}: \beta<\alpha\right\}\right|=|\alpha|<\kappa$, by $\kappa$-saturation we can pick $f_{\alpha} \in \bigcap_{\beta<\alpha} F_{\beta}$. Clearly $f_{\alpha}$ satisfies the conditions 1 and 2 .

The union $f=\bigcup_{\alpha<\kappa} f_{\alpha}$ yields the desired order-isomorphism.

Corollary 3.4. Independently of the value of $\operatorname{cof} \theta=\operatorname{cof} \theta^{*}$,

$$
\mu=\rho=\gamma \theta \Longrightarrow \theta=\theta^{*} .
$$

Proof. By Lemma 3.3, the models of (ii) Theorem 3.2 with $\kappa_{1}=\kappa_{2}=\kappa$ satisfy the equality $\mu=\rho$ together with $\exists x \exists y\left(\theta_{x} \neq \theta_{y}\right)$, which contradicts $\theta=\theta^{*}$, by Theorem 1.3 .

The next theorem will yield that also the second implication of Corollary 1.5 cannot be reversed. In the proof of the theorem we shall need the notion of type of a gap. Therefore we recall the following definition

Definition 3.5. Let $(X, Y)$ be a partition of the ordered set $(S,<)$.

- The partition $(X, Y)$ is a gap of $S$ if $X$ has no greatest element, $Y$ has no least element, and $X<Y$ (i.e. $x<y$ for all $x \in X$ and $y \in Y$ ).

- The gap $(X, Y)$ has type $(\kappa, \zeta)$ if $\operatorname{cof} X=\kappa$ and $\operatorname{cof} Y^{*}=\zeta$.

- The gap $(X, Y)$ is generated by $A \subset S$ if

$$
X=\{x \in S \mid x \leq a \text { for some } a \in A\} .
$$

Theorem 3.6. Let $\kappa$ be a regular cardinal and assume $\kappa>2^{\zeta}$. Then there exist $\zeta^{+}$-saturated nonstandard models where $\operatorname{cof} \theta=\operatorname{cof} \theta^{*}=\kappa$, but $\rho \neq \mu$. 
Proof. Let $\mathcal{G}$ be a $\left(2^{\zeta}\right)^{+}$-saturated model where $\operatorname{cof} \theta=\kappa$. (Such a model exists for $\kappa>2^{\zeta}$.) Let $D$ be a countably incomplete $\zeta^{+}$-good ultrafilter over $\zeta$. Let $\mathcal{D}$ be the $\kappa$-sequence constantly equal to $(D, \zeta)$. We claim that the model $\mathcal{U}=\mathcal{U}(\mathcal{G}, \kappa, \mathcal{D})$ satisfies the thesis.

By Lemma 2.6, the model $\mathcal{U}$ is $\zeta^{+}$-saturated, and satisfies $\operatorname{cof} \theta^{*}=\operatorname{cof} \theta=\kappa$.

We are left to show that $\rho \neq \mu$ in $\mathcal{U}$. By contradiction, assume that there is an order-isomorphism $\Psi:{ }^{*} \mathbb{R} \rightarrow M$ between the nonstandard real line and the monad of zero in $\mathcal{U}$. In the following we denote by ${ }^{*} \mathbb{R}_{\alpha}$ and $M_{\alpha}$ the hyperreal line and the monad in $\mathcal{U}_{\alpha}$, respectively. Now ${ }^{*} \mathbb{R}_{0}$ is cofinal in ${ }^{*} \mathbb{R}$ and, for every $\alpha<\kappa, M_{\alpha}$ is not cofinal in $M_{\alpha+1}$, by Lemmata 2.4 and 2.6. Therefore we can pick increasing $\zeta^{+}$-sequences $\left\{x_{\alpha} \mid \alpha<\zeta^{+}\right\}$in ${ }^{*} \mathbb{R}_{0}$ and $\left\{\beta_{\alpha} \mid \alpha<\zeta^{+}\right\}$in $\kappa$ such that

$$
\Psi\left(x_{\alpha+1}\right)=y_{\alpha+1} \in M_{\beta_{\alpha+1}} \text { is greater than all elements of } M_{\beta_{\alpha}}
$$

In $* \mathbb{R}_{0}$ the $\zeta^{+}$-sequence $\left\{x_{\alpha} \mid \alpha<\zeta^{+}\right\}$generates a gap $\left(X, X^{\prime}\right)$ of type $\left(\zeta^{+}, \xi\right)$, with $\xi>2^{\zeta}$, by $\left(2^{\zeta}\right)^{+}$-saturation. Since $\zeta<\zeta^{+}<\xi$, the $\zeta^{+}$-sequence $\left\{x_{\alpha} \mid \alpha<\zeta^{+}\right\}$ generates a gap of the same type in the iterated ultrapower $\mathcal{U}$ as well.

On the other hand, the $\zeta^{+}$-sequence $\left\{y_{\alpha} \mid \alpha<\zeta^{+}\right\}$is cofinal in $M_{\beta}$, where $\beta=\sup \left\{\beta_{\alpha} \mid \alpha<\zeta^{+}\right\}<\kappa$. So it generates a gap of type $\left(\zeta^{+}, \omega\right)$ on ${ }^{*} \mathbb{R}_{\beta}$ and cosequently one of type $\left(\zeta^{+}, \eta\right)$ with $\zeta<\eta \leq 2^{\zeta}$ in ${ }^{*} \mathbb{R}_{\beta+1}$. The latter type remains unchanged up to $* \mathbb{R}$, by the argument above. This is a contradiction because, by order-isomorphism, both gaps must have the same type. ${ }^{4}$

We are now ready to deal with the converse of the second implication of Corollary 1.5

\section{Corollary 3.7.}

(1) If $\operatorname{cof} \theta=\operatorname{cof} \theta^{*}=\omega$, then $\mu=\rho=\gamma \theta$.

(2) If $\operatorname{cof} \theta>2^{\omega}$, then $\operatorname{cof} \theta=\operatorname{cof} \theta^{*} \not \mu=\rho$.

Proof. Point 1 follows from Lemma 3.3, since all nonstandard models are $\omega$-saturated. Point 2 is an immediate consequence of the theorem above for $\zeta=\omega$.

\section{FinAL REMARKS}

The consistency and the mutual relations of the order-theoretic properties $(A 1)-$ $(A 5)$ and $(B 1)-(B 5)$ of Section 1 , have been studied by several authors since the problem has been explicitly posed in the paper of E. Zakon [30]. We recall here only the main achievements which are known to us.

The existence of models where cofinality and coinitiality of the infinites can be arbitrarily fixed has been first proved by K. Potthoff in [23], where construction techniques substantially equivalent to those of Section 2 are developed.

The consistency of $\theta=\theta^{*}$ has been first proved, in [17] and [29], only for fully saturated models, hence under heavy set-theoretic assumptions and with strong limitations on size and cofinality of $\theta$. It is worth mentioning that Lemma 3.2 of [17] claims that $\operatorname{cof} \theta=\operatorname{cof} \theta^{*}$ is equivalent to $\rho=\mu$, contradicting Point 2 of our Corollary 3.7. But the purported inductive proof works only for successor steps,

\footnotetext{
${ }^{4}$ The argument of this proof has been suggested to the first author by Renling Jin in a personal communication.
} 
and so it proves in fact only Point 1 of our corollary. Notice that this fault affects also the proofs of some true equivalences among the properties $(B 1)-(B 5)$, which are derived there from this false lemma.

The first general constructions allowing for symmetric infinites with arbitrary cofinalities can be found in [9], where also several implications among the properties $(A 1)-(A 5),(B 1)-(B 5)$ are proved (see also [7], where Theorems 1.3, 3.1 and 3.2 of the present paper are stated without proof).

We think that the main questions concerning the above order-theoretic properties which remain open are the following: ${ }^{5}$

(1) Are there models where $\theta=\theta^{*} \neq \rho$ ?

(2) Are there models where $\omega<\operatorname{cof} \theta=\operatorname{cof} \theta^{*} \leq 2^{\omega}$ and $\mu \neq \rho$ ?

(3) Are there models where $\forall x \forall y\left(\theta_{x}=\theta_{y}\right)$ but $\theta \neq \theta^{*}$ ?

We conjecture that the answers to these three questions are all in the positive. For instance, it seems that the model $\mathcal{V}(\mathcal{U}(\mathcal{G}, \kappa, \mathcal{D}), \omega, \mathcal{E})$, where $\mathcal{U}(\mathcal{G}, \kappa, \mathcal{D})$ is the model in the proof of Theorem 3.1 and $\mathcal{E}$ is constantly equal to some nonprincipal ultrafilter over $\omega$, should satisfy

$$
\forall x \forall y\left(\theta_{x}=\theta_{y}\right) \text { together with } \operatorname{cof} \theta^{*}=\kappa \text { and } \operatorname{cof} \theta=\omega .
$$

Another interesting topic, stemming from [30] and not considered here, is the study of the types of the gaps that can occur in the hyperreal line and their connections with the order-type $\theta$. This topic is strictly connected to properties of the $Q$-topology and of other natural non-Hausdorff topologies of the hyperreal line. Several interesting results in this direction can be found in the papers cited above, and, among others, also in [16],[9],[21],[13],[14],[27]. (We are considering these questions in a paper under construction.)

In this paper, all models have been explicitly defined by means of the ultrapower construction. The idea of using iterations of ultrapowers and internal ultrapowers has been used extensively in the literature. See e.g. the excellent paper [21] where these techniques are exploited in order to solve the difficult problem of finding sufficiently saturated models where the hyperreal line is Scott complete.

After the pioneering article of Henson [12], where the Isomorphism Property was introduced, a new interesting approach has been developed, namely that of isolating "special" principles which hold in suitable classes of models. Among them, we single out the the Special Model Axiom of Ross [26], the Resplendency Property of Jin and Shelah [15], and the recent combinatorial $\Delta$-principles of the first author and Hrbàcek $[2,3]$. These "special" properties have been showed fruitful to show the consistency of several properties in nonstandard analysis, and we think that they could be successfully applied also to the topics treated in this paper.

\footnotetext{
${ }^{5}$ Notice that in this paper we are considering only results that are provable in ZFC. The relative consistency of $\theta=\theta^{*} \neq \rho$ with ZFC+ "there exists a huge cardinal" is a consequence of the consistency of Foreman's nonregular ultrafilter $\mathcal{D}$ over $\omega_{2}$, see [6]. In fact the ultrapowers $\omega_{\mathcal{D}}^{\omega_{2}}$ and $\omega_{1} \underset{\mathcal{D}}{\omega_{2}}$ have sizes $\aleph_{1}$ and $\aleph_{2}$, respectively. Therefore, in the corresponding nonstandard model, $\theta=\theta^{*}$ holds because $\theta$ is a $\aleph_{1}$-saturated order-type of size $\aleph_{1}$. On the other hand $\theta \neq \rho$ since $|\rho| \geq \aleph_{2}$.
} 
In particular, the $\Delta$-principles have the advantage of being formulated in purely combinatorial terms, and they can be directly applied without any use of modeltheoretic constructions, such as ultrapowers. For instance, by simple combinatorial arguments, it is shown in [3] that $\Delta_{2}$ proves $\theta=\theta^{*}$.

\section{REFERENCES}

[1] C.C. Chang and H.J. Keisler, Model Theory (3rd edition), North-Holland, Amsterdam, 1990.

[2] M. Di Nasso, The generic filter property in nonstandard analysis, Ann. of Pure and Appl. Logic 111 (2001), 23-37.

[3] M. Di Nasso and K. Hrbàček, Combinatorial principles in nonstandard analysis, Ann. of Pure and Appl. Logic, to appear.

[4] M. Di Nasso and Y. Zhang, Nonstandard analysis and an application to the symmetric group on natural numbers, this volume.

[5] R. Engelking, General Topology, Polish Scientific Publishers, Warszawa 1977.

[6] M. Foreman, An $\aleph_{1}$-dense ideal on $\aleph_{2}$, Isr. J. Math. 108 (1998), 253-290.

[7] M. Forti, On the order structure of the nonstandard real axis, in Atti degli incontri di Logica Matematica, vol.3 (R. Ferro and A. Zanardo, eds.), Padova 1987, 199-205.

[8] M. Forti, On the order structure of the nonstandard real axis (abstract), J. of Symb. Logic 52 (1987), 317-318.

[9] M. Forti and F. Honsell, Sull'ordinamento dei numeri reali non-standard (italian), Atti Acc. Naz. Lincei Rend. Cl. Sci. Fis. Mat. Nat. S. 874 (1985), 1-8.

[10] M. Forti and F. Honsell, Choice Principles in Hyperuniverses, Ann. Pure Appl. Logic 77 (1996), 35-52.

[11] W.S. Hatcher and C. Laflamme, On the order structure of the hyperreal line, Zeitschr. fur Math. Logik und Grund. der Math. 29 (1983), 197-202.

[12] C.W. Henson, The isomorphism property in nonstandard analysis and its use in the theory of Banach space, J. of Symb. Logic 39 (1974), 717-731.

[13] R. Jin, Cuts in hyperfinite time lines, J. of Symb. Logic 57 (1992), 522-527.

[14] R. Jin, U-monad topologies of hyperfinite time lines, J. of Symb. Logic 57 (1992), 534-539.

[15] R. Jin and S. Shelah, The strength of the isomorphism property, J. of Symb. Logic 59 (1994), $292-301$.

[16] S. Kamo, Non-standard real number systems with regular gaps, Tsukuba J. Math. 5 (1981), $21-24$.

[17] S. Kamo, Nonstandard natural number systems, J. of Symb. Logic 46 (1981), 365-376.

[18] V. Kanovei, M. Reeken, Mathematics in a nonstandard world. I, Math. Jap. 45 (1997), 369-408.

[19] H.J. Keisler, Monotone complete fields, in Victoria Symposium on Nonstandard Analysis (A. Hurd, P. Loeb, eds.), Berlin 1974, 113-115.

[20] H.J. Keisler, Foundations of Infinitesimal Calculus, Prindle, Weber and Schmidt, Boston, 1976.

[21] H.J. Keisler and J.H. Schmerl, Making the hyperreal line both saturated and complete, J. of Symb. Logic 56 (1991), 1016-1025.

[22] E. Nelson, Internal Set Theory; a new approach to nonstandard analysis, Bull. Amer. Math. Soc. 83 (1977), 1165-1198.

[23] K. Potthoff, Ordnungseigenschaften von nichstandardmodellen, in Theory of Sets and Topology (in honour of Felix Hausdorff) (G. Asser, J. Flaschmeyer, and W. Rinow, eds.), Berlin 1972, 403-426.

[24] M. Prokhorova, External set properties in IST (abstract), Logic Colloquium 2001, Volume of abstracts, Vienna 2001.

[25] A. Robinson, Non-standard Analysis, North Holland, Amsterdam 1966.

[26] D. Ross, The special model axiom in nonstandard analysis, J. of Symb. Logic 55 (1990), 1233-1242.

[27] S. Shelah, Vive la différence II. The Ax-Kochen isomorphism theorem, Israel J. of Math. 85 (1994), 351-390.

[28] N. Vakil, Nonstandard topologies with bases that consist only of standard sets, Proc. Amer. Math. Soc. 129 (2001), 2075-2083. 
[29] S. Yang, D. Wang and J. Wang, On the order structure of the $\omega$-regular extension hyperreal numbers (chinese), Acta Math. Sin. 23 (1980), 658-667.

[30] E. Zakon, On the order structure of the nonstandard real axis, in Applications of Model Theory to Algebra, Analysis and Probability (W.A.J. Luxemburg, ed.), New York 1969, 195227.

Dipartimento di Matematica Applicata "U. Dini", Università di Pisa, Italy.

E-mail address: dinasso@dma.unipi.it

Dipartimento di Matematica Applicata "U. Dini", Università di Pisa, Italy.

E-mail address: forti@dma.unipi.it 